\title{
Sleep Disorder Diagnosis through Frequency based EEG Energy Level Patterns
}

\author{
Shivam Tiwari ${ }^{1}$, Deepak Arora ${ }^{2}$, PuneetSharma ${ }^{3}$, Barkha Bhardwaj ${ }^{4}$ \\ 1, 2,3,4 Department of Computer Science and Engineering, Amity University, Lucknow Campus, India \\ 1'shivam.tiwari.info@gmail.com, 2deepakarorainbox@gmail.com,3 puneetgrandmaster@gmail.com, \\ ${ }^{4} 123$ barkhabhardwaj@gmail.com
}

\begin{abstract}
EEG data has proved to reflect the activities of brain over all the section with respect to human activities. They are useful in both cases that is in awakening states and in sleep stage. Most of the brain disease is due to the deterioration of brain cells. In this research work, EEG data is used to generate some frequency based energy level features for RBD patients and normal persons. After experimentation, It is found that percentage of energy levels may be considered as parameter to distinguish healthy and defected EEG data due to sleep disorder. During this study, it is also found that $\mathrm{S} 0$ is proved to be helpful in diagnosis of RBD sleep disorder because in this stage the percentage energy level is found to be low for alpha waves for patients having sleep disorder. The results shows that the average percentage energy level in RBD patient is 0.1139 while in normal cases it is 0.1530 during alpha activity.
\end{abstract}

Key words: EEG, Sleep disorder, Pattern Recognition, and Data Mining.

\section{INTRODUCTION}

In the past twenty years abundance of research work is published on new proposals and algorithms on biomedical signals like EEG, ECG etc. which supports the use of power estimation in frequency domain for several disorders detection and their diagnosis. One of the article published by Penzel et al., which demonstrated the use of single-lead ECG for diagnosis of sleep disorders [10]. After this many literature is published remark ably on such king of ECG data for simplifying the use of instruments for data recording and reducing computation complexity. All of such kind of research work focused on extracting the patterns or called features. The selection of the optimal parameter of calculations is universally accepted in numerous kinds of advanced machine learning techniques. Heart-rate-variability (HRV) [10], consecutive heart beat (RR) time-period, and respiratory data records extremely used for finding outsets of the feature to use in pattern recognition and machine learning [11]. These kind of biomedical data features used in modern pattern recognition application in diagnosis of several human disorders and disease using variety of domains of time and frequency [1213]. Appropriate features are to be significantly sorted out for simple and fast statistical equations [13] or evaluation methods $[11,14]$. One of the approach is principal component based extraction of feature that helps to reduce dimensions and improve detection accuracy of diagnosis for specific disease.
In this research work, focus is given on diagnosis of sleep disorders. Sleep is very crucial activity because in a lifespan time sleep covers 1/3 part of the lifetime. Sleep is important like other activities such as eating, drinking and breathing. In the moments under sleep body and brain repair itself by interacting through hormones, muscles neurons and memory. sleep disorder occurs when someone cannot sleep properly it results in lose function of the body organs and muscles [15]. The common benefit of the sleep covers physical, emotional and psychological impacts. Improper sleep damages us in physical, emotional and psychological aspects. Nearly 84 types of sleep disorders have been observed like narcolepsy, insomnia, sleep-apnoea and restless-leg-syndrome etc. [1]. Sleep Apnoea (SA) represents the stage when pause in the breathing occurs during sleep. Pause of the breath called as apnoea and it varies with time period and repetition. The problem in breath while someone sleep is symptom of hypopnea [2]. Sleep disorder presently divided as Obstructive Sleep Apnoea (OSA), very common (2\% to $4 \%$ adult population whereas $1 \%$ to $3 \%$ in children [3]. It occurs due to disturbance in respiration in throat airway. Another category of sleep disorder is Central Sleep Apnoea (CSA) occurs due to inhibited respiration drive [4]. Sleeping disorders do not occur only in the intervals of night. In day-time from excessive sleepiness, loose type of mind concentration, high level of the head-ache etc. occurs [3]. In night intervals duration due to the effect of sleep disorder, choking, noise, sweat etc. can be observed. OSA is found in abundance in middle age or elders and causes obesity [3] in most situation. It is recorded that 70 billion dollars loss, 11.1 billion in damages and 980 deaths in each year [5]. Most cases go undiagnosed because of the inconvenience, expenses and unavailability of testing. Testing is inconvenient to the patient because it requires them to spend the night away from their bed causing discomfort. It is expensive because overnight stay in hospital because of the requirements of machines and expert level of technicians. Testing is also widely unavailable due to sleep centers operating at full capacity and those on the waiting list can be untreated for an additional 6months.Testing includes polysomnography, airflow records of subject, respiration, oxygen saturation, body position, EEG, electrocardiogram ECG [6].

\section{RELATED WORK}

In 2018, research have been turned with a gold standard classification accuracy by picking up human sleep stages, the use of Heart Rate Variability (HRV) functions based on 
electrocardiogram (ECG) sign. The proposed technique is the combination of Extreme Learning Machine (ELM) and Particle Swarm Optimization (PSO) for characteristic selection and hidden node range dedication. The combination of ELM and PSO produces imply of trying out accuracy of $82.1 \%, 76.77 \%$, $71.52 \%$, and $62.66 \%$ for $2,3,4$, and 6 range of lessons respectively. This study additionally offers assessment to ELM and Support Vector Machine (SVM) strategies, whose testing accuracy is lower than the combination of ELM and PSO. Based on the outcomes, it is concluded that the addition of PSO method is capable giving overall great performance [8]. This research proposed a unique method for automatically detecting sleep-disordered breathing (SDB) events the usage of a recurrent neural community (RNN) to investigate nocturnal electrocardiogram (ECG) recordings. A deep RNN version have been designed which comprises of six stacked recurrent layers for the automated detection of SDB occasions. The proposed deep RNN version makes use of lengthy short-time period reminiscence (LSTM) and a gated-recurrent unit (GRU). To compare the performance of the proposed RNN technique, 92 SDB sufferers had been enrolled. Single-lead ECG recordings were measured for a mean 7.2-h length and segmented into 10-s activities. The dataset comprised a training dataset (545 activities) from 74 patients and a dataset (17,157 activities) from 18 patients. The proposed approach finished high overall performance with an F1-rating of ninetyeight, $0 \%$ for LSTM and $99.0 \%$ for GRU. The effects show advancement in overall performance over conventional methods. The proposed technique can be used as a particular screening and diagnosing device for sufferers with SDB problems [9]. Different sleep related diseases affect different body parts prominently. Breathing-related sleep disorders are explained through polysomnography reports. In this type of disorder, breathlessness has been seen for 10 seconds. These disorders are called sleep apnea. According to scientific research, sleep apnea disease can be the reason of heart related disorders. Confirmation of such diseases is done by periodic observation of readings of several polysomnography channels.

All the methods used to confirm sleep disorders are very expensive and time consuming. Periodically polisomnography check up in diseases like sleep apnea puts financial burden on patients. In order to make such time consuming and expensive tests simple and less expensive, ECG signals were studied. Research confirms that ECG readings are capable of confirming diseases like sleep apnea. Tunable-Q wavelet Transfom (TQWT) techniques were used for better understanding of ECG readings. The TQWT method is able to decompose the ECG readings in numeric form of bandwidth and make it understandable. The Centered Correntropy (CCES) is calculated from the results obtained by TQWT Method. Readings of CCES are tested using the line acting classifier on the obtained visualized waveform results. In this experiment, sleep apnea was tested with $92.7 \%, 90.9 \%$ and $93.9 \%$ accuracy by using random forest machine learning algorithm in 3 different cases based on ECG records[10].karthik offered a suitable and efficient implementation of a characteristic extraction algorithm (Pan Tompkins Algorithm) on electrocardiography (ECG) indicators, for detection and category of four cardiac sicknesses: Sleep Apnea, Arrhythmia, Supraventricular Arrhythmia and Long Term Atrial Fibrillation (AF) and differentiating them from the regular heart beat by the use of pan Tompkins RR detection observed through feature extraction for classification. The study additionally presents a brand new technique closer to signal category using the prevailing neural networks classifiers [10]. A wise feature selection from minute-to-minute electrocardiogram (ECG) sign is a challenging challenge for plenty motives, but largely because of correct detection of scientific problems, together with the sleep apnea. In 2019, the ECG sign was modeled with a purpose to achieve the Heart Rate Variability (HRV) and the ECG-Derived Respiration (EDR). Selected capabilities strategies used for benchmark with distinctive classifiers including Artificial Neural Networks (ANN) and Support Vector Machine (SVM), among others. The results shows firstrate accuracy as $82.12 \%$, with a sensitivity and specificity of $88.41 \%$ and $72.29 \%$, respectively. In addition, experiments found out that a smart characteristic selection may also enhance the machine accuracy. Therefore, the proposed version found out to be dependable and easier opportunity to classical answers for the sleep apnea detection, for instance based on the Polysomnography. [11]

\section{METHODOLOGY}

Sleep process is responsible for both physical and mental health. In 1913 first analysis using close examination of sleep activity was reported. In 1920s research was accomplished on sleep and wake stage along with the circadian signals. Sleep data based characteristics were observed to identify the sleep deprivation. Cyclic behaviour in sleep process was found in 1955 to justify the significant relationship in REM sleeping and dream activity [12].

It was proved that major part of dreams occur in REM sleep stage process and remaining observed in NREM sleep duration activities. It is easier to get awaken in REM dreams as compared to the dreams during NREM phase. Recalling of dreams is easy if a person is awakened just on start of REM dreams as compared to next morning awakening. REM dreams are generally observed to be very unrealistic as well as bizarre. Dream recalling is observed which to be occur partially when awakening is just in NREM stage of dreaming and such dream moments are somewhat realistic in nature. Sleep stages mostly covers the visual sensations along with some extent of auditory sensations [13]. The smell and taste sense are observed to be occurring in least proportion. Dream behaviour also involves movements in sleep called as REM parasomnia and treated as REM sleep behaviour disorder (RBD). Sleep is divided in a cyclic pattern in between N-REM and REM sleep stages [1-6]. In the moments of Non- REM sleep is classified, sleep behaviour of human beings is observed to be changing from birth to old age with the passage of time. In sleep stage 0 (awake) eyes are unlock and the EEG is unreliable and very significantly with low voltage value (beta waves), eyeball movement are slow and the EEG data signal frequency is in between 6 to $8 \mathrm{~Hz}$. The S1 stage of sleep consist of alpha waves and occurs during the drowsiness activity. In the process of Sleep cycle stage 2, light sleep occurs with very slow eye movements on the onset of getting stop and brain waves signal gradually getting slower. In this stage, sleep spindles are also observed to be started and EEG data value is found average and the range of data frequency exist in between $4-7 \mathrm{~Hz}$. In the duration observed within occurrence of stage 3 sleep activity moments also known as deep sleep the brain waves rhythms are extremely slow and called as delta waveforms 
starts to be observed. These waves are interpreted as smaller but faster waveforms. EEG data at this zone of sleep activity possess frequency $1-3 \mathrm{~Hz}$ and strength is high. In the sleep cycles of duration occurring in stage 4 also known as deep sleep exhibiting the slow wave the brain initializes the generation of delta waves. In this zone the value of EEG is high and the frequency is below the value of $2 \mathrm{~Hz}$. In this stage eye movements are found to be rapid and momentarily muscular moves are associated. Theta wave is common in such sleep stage cycle moments. To better analysis of waveform of REM sleep pattern, it is converted in Power spectrum. For Power conversion by using Welch method. With Welch method, large wave pattern is converted to imbricate.

Following three steps used for this, are as under:

(i) Wave pattern is divided in $\mathrm{K}$ segments by considering the original wave pattern length as $\mathrm{L}$.

(ii) Window created in step I, enforced to each section

(iii) Take average of $\mathrm{K}$ periodograms for wave pattern

$$
P_{w}\left(e^{j \omega}\right)=\frac{1}{k} \sum_{k=1}^{K} P x^{(k)}\left(e^{j \omega}\right) .
$$

Where,

$$
X_{P_{x}^{(k)}}=\frac{1}{N} \sum_{n=0}^{L-1}\left|w_{(n)} x^{(k)}(n) e^{-j \omega n}\right| \ldots \ldots \ldots
$$

Above method is applied as per following phases:

Phase 1: Import EEG data file in desired format shows in Fig 1.

Phase 2: EEG data is extracted after downloading 30 to 60 seconds records of sleep movements in different moments of sleep stages of different channels like ROC-LOC, C4-P4 etc shows in Fig 2.

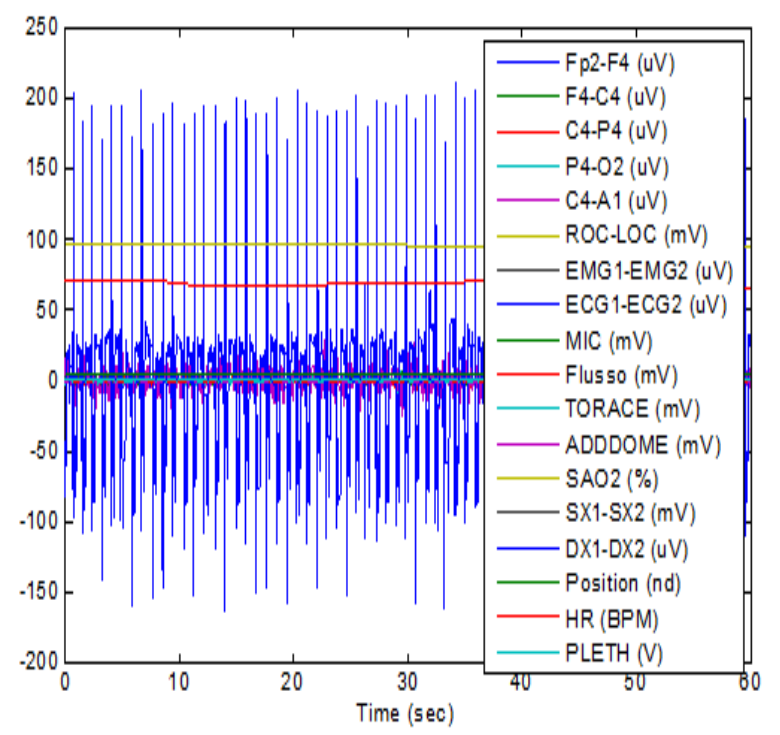

Figure 1: Extracted EEG data signal

Phase 3: Removal of mean value, component with zero frequency obtained by FFT algorithm is the mean value of data, which is subtracted from the data to bring the all data at similar mean value and bring in the same in range.

Phase 4: After removing the mean value to bring all data in common range the unwanted frequency and noise is subtracted. This is generally of higher frequency above than the EEG data frequency nearly above the $40 \mathrm{~Hz}$. For this purpose, filter are used that passes low frequency data and stops high frequency values, which are generally anomaly in terms of noise, fluctuations or error. The resultant data after filtering has following benefits:

(i) No high frequency distortion

(ii) Only data within EEG frequency level is obtained
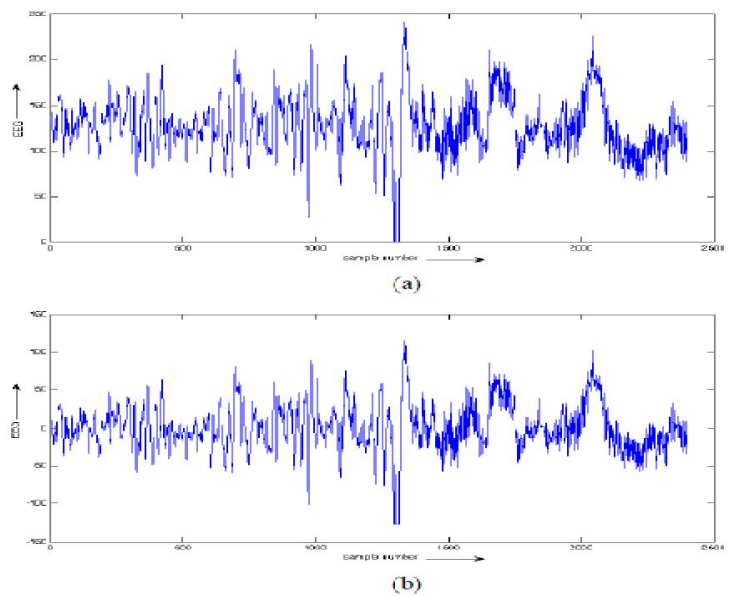

Figure 2 (a): EEG data before (b) and after removing mean value

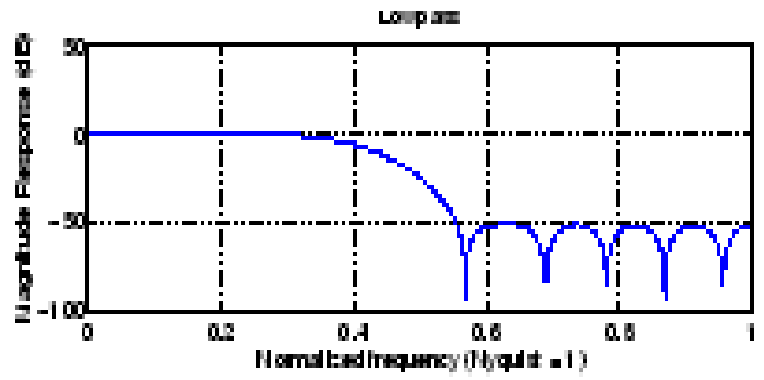

Figure 3: Filter Data Plot after removing high frequency distortions and anomaly

Phase 5: Power Calculation, the power estimation is very crucial to figure out the features of data abnormality in defective cases shows in Fig 3. Welch algorithm is used to calculate the frequency component power by using period gram after applying the Fast Fourier Transform algorithm. The periodogram basically uses the formulae for autocorrelation of fix length of a data after clipping or segmenting in equal parts. It give simple approach to provide results with very high accuracy.

The periodogram estimate of the frequency components power of fixed length-L of any data segment $x_{\mathrm{L}}[n]$ is

$$
P_{x x}(f)=\frac{1}{L F_{s}} \sum_{n=0}^{L-1} x_{L(n) e^{-j 2 \pi f_{n} / F_{y}} \ldots}
$$


$\mathrm{F}_{\mathrm{s}}$ stands for the sampling frequency.

$$
f_{n}=\frac{k F_{s}}{N} k=0,1 \ldots, N-1
$$

Phase 6: Area covered by plot of power value under delta, theta, alpha, gamma frequency range is found through Trapezoidal rule. Theta $(\theta)$ frequency 4 to $8 \mathrm{~Hz}$, delta $(\delta)$ frequency is considered from 0.5 to $4 \mathrm{~Hz}$, alpha $(\alpha)$ frequency range 8 to $13 \mathrm{~Hz}$ and finally the significant one beta $(\beta)$ wave frequency is considered as 13 to $30 \mathrm{~Hz}$.

Phase 7: After getting the Power under each brain data waves the ratio of these values are intended by isolating the average power of each sleep wave frequency range by the associated average power for complete bands.

\section{RESULT AND DISCUSSION}

Figure 4 shows various signals consisted by our biomedical signals. All signals are obtained by performing signal data extraction of data file named as n1_edfm.mat.It shows the waveform of EEG data records at different channels like voltage data in between ROC and LOC, F2-F4 etc. All the data is in microvolt and stored in the text files. These files are imported to MATLAB and the data is clipped into small parts called as segments.

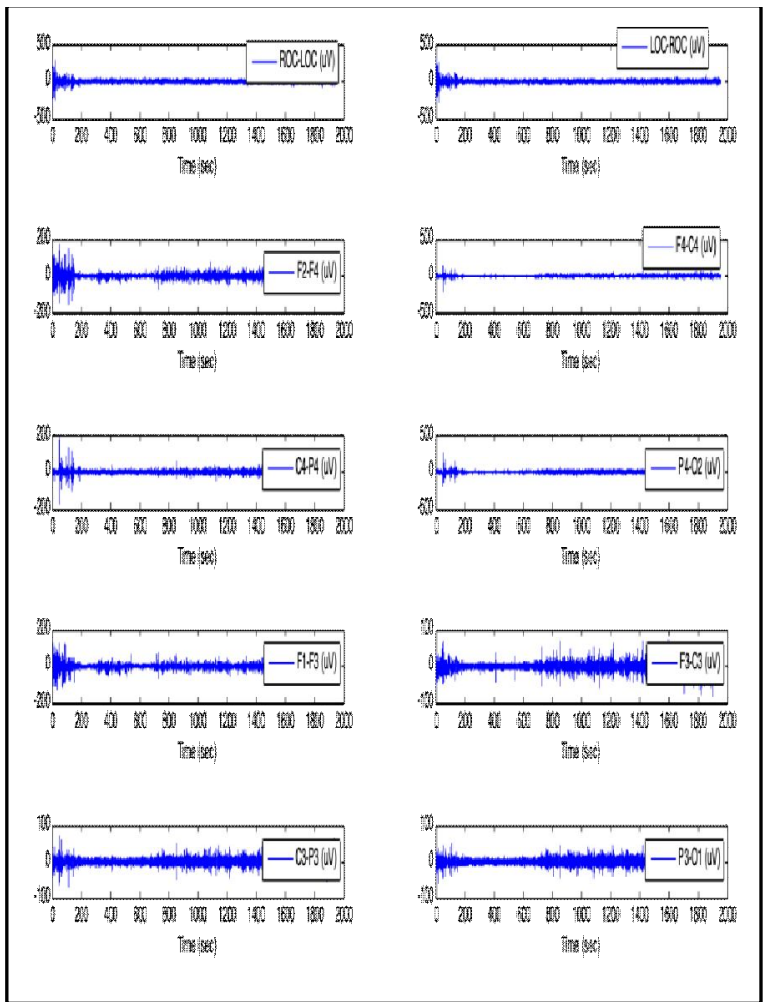

Figure 4: Waveform for data by recorded signals 'n1_edfm.mat' varying from signal 1 to 10

All previous figures 4 indicates the clipped part of various sleep stages during S0 and REM moments taken from the normal and pathological cases. These plots can further be analyzed for presence of any variation in magnitude and wave shape patterns of waves in both different cases. Hence normal and pathological cases can express some differences in there features but it cannot be proved directly because EEG signals are multivariable signals and there pattern depends upon mental status of the subject.

Figure 4 was the complete data plot for EEG recordings of $200 \mathrm{sec}$ with respect to time. This data is segmented for small interval of and shown in the figure 5.These small segments are of $2 \mathrm{sec}$ of time interval. These segments are sorted out in separate database as per the different sleep stages varying from S1 to S4. Same process of segmentation and sleep stage wise separation task is performed for all the subjects belonging to normal cases and cases belonging to sleep disorders.
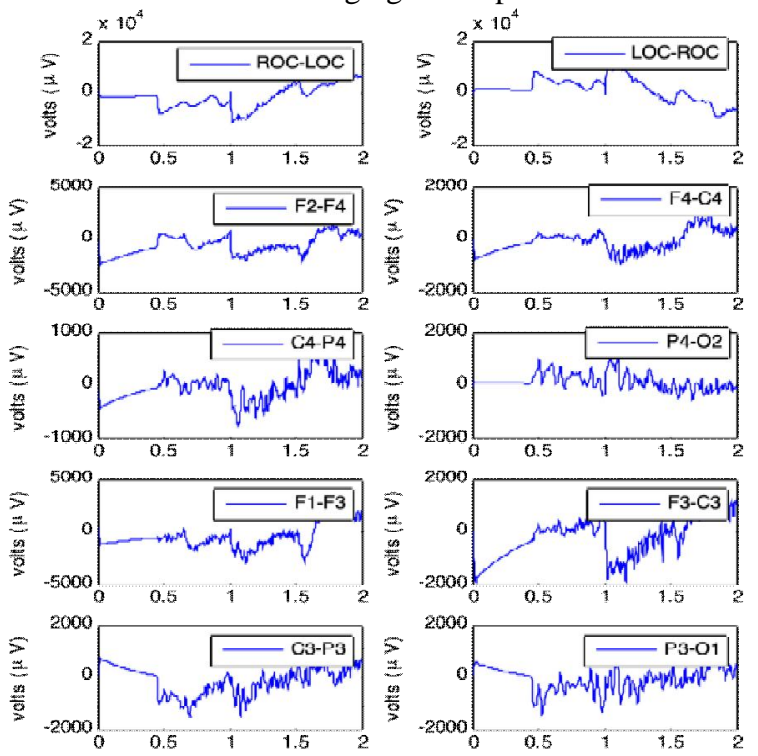

Figure 5: Segmented data for all the channels for interval of $2 \mathrm{sec}$

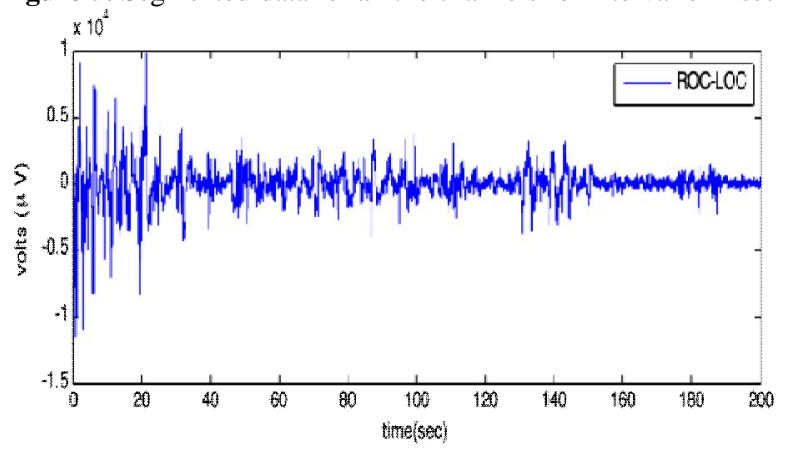

Figure 6: Individual EEG signal of ROC-LOC channel

For each person the data records are collection of 20 channels data. Some of the channels are shown in previous figures (4 and 5).In the figure 6 one of the pot of collective EEG data set is shown to represent a specific channel. This data is passed through processing steps to generate featured patterns that help to perform diagnosis of a particular sleep disorder. As the data is taken it is segmented out individually and shown in figure 7. This given segment is clipped out on the time intervals in which the patient is under going through the rapid eye movement (REM) sleep stage. In a similar way all the segments of normal and abnormal cases are clipped out as per the available time interval of particular stages. 


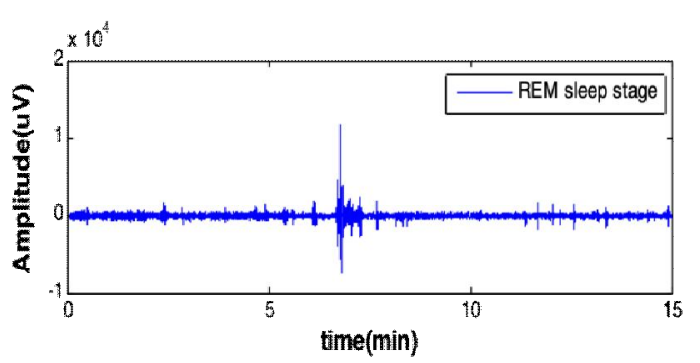

Figure 7: Segmented data plot of REM stage clipped from data shown in figure 6

All the segmented parts of short duration for sleep stages during S0, S1, S2, S3 and REM intervals are collected for multiple normal and pathological EEG datasets. The analysis of these plots in terms if variations in the magnitude and wave shape patterns in both different cases are very random and fast. Hence by visual inspection even experts can not definitely predict that whether it belongs to normal or pathological cases. But to simply the wave pattern it is necessary to remove noise from data pattern as shown in figure 8 .

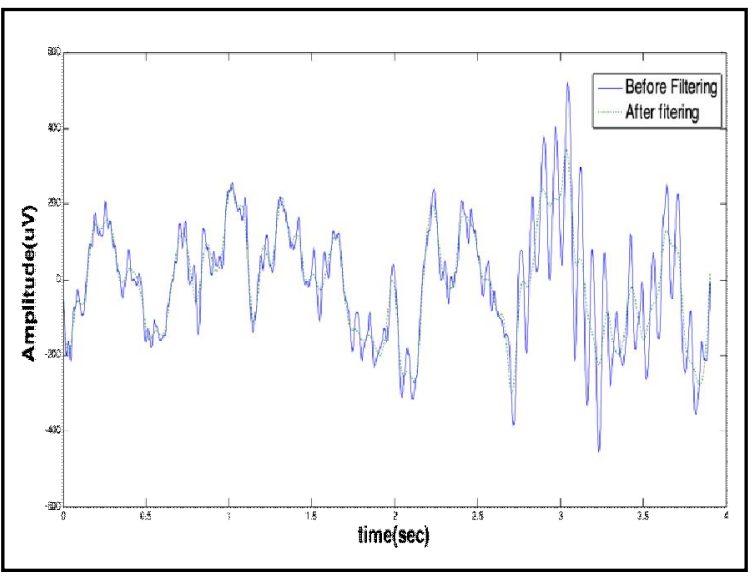

Figure 8: Segmented data with noise (_ $)$ and after removing the noise (---)

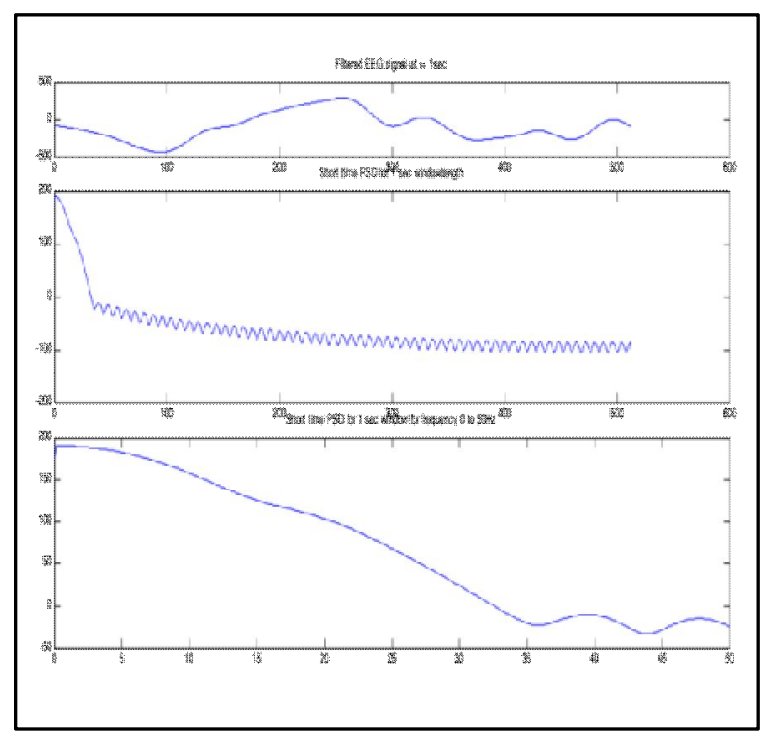

Figure 9: PSD of S0 Stage for Normal Cases without any sleep disorder
After removing the unwanted noise and distortion the data plots become smooth. These data records for all the cases of different sleep stages are used to calculate the Fourier transform of records. The transform data distributes the signal with respect to frequency. It displays the strength (magnitude) in terms of voltage at different frequencies. This strength is further used to calculate the respective power or energy of different frequency. The graph in between signal power at each frequency is called as power spectrum density (PSD).

In the figure 9 the power with respect to available frequency (middle) is shown for EEG data segment (top). Here the frequency is from 0 to $50 \mathrm{~Hz}$ with 512 samples.

Table 1: REM stage analysis of RBD patient EEG data in terms of Energy Level

\begin{tabular}{|l|l|l|l|}
\hline & Case 1 & Case 2 & Case 3 \\
\hline $\begin{array}{l}\text { Delta } \\
\text { Wave }\end{array}$ & 0.55049 & 0.59427 & 0.61224 \\
\hline $\begin{array}{l}\text { Theta } \\
\text { Wave }\end{array}$ & 0.31859 & 0.30304 & 0.28745 \\
\hline $\begin{array}{l}\text { Alpha } \\
\text { Wave }\end{array}$ & 0.12593 & 0.098331 & 0.095321 \\
\hline Beta Wave & 0.0049856 & 0.0043566 & 0.0049866 \\
\hline
\end{tabular}

Energy level of beta wave is lowest in both cases. The percent energy level of delta and theta are high always for both tables (Table 1-2). Alpha wave is always in medium level energy value. Hence the REM stage is not helpful in classifying the normal and RBG infected persons.

Table 2: REM stage analysis of Normal Person EEG data in terms of Energy Level

\begin{tabular}{|l|l|l|l|l|l|}
\hline & $\begin{array}{l}\text { Case } \\
\mathbf{1}\end{array}$ & $\begin{array}{l}\text { Case } \\
\mathbf{2}\end{array}$ & Case 3 & Case 4 & Case 5 \\
\hline Delta Wave & $\begin{array}{l}0.701 \\
85\end{array}$ & $\begin{array}{l}0.412 \\
51\end{array}$ & $\begin{array}{l}0.5191 \\
9\end{array}$ & $\begin{array}{l}0.6018 \\
6\end{array}$ & 0.3634 \\
\hline Theta Wave & $\begin{array}{l}0.245 \\
21\end{array}$ & $\begin{array}{l}0.317 \\
05\end{array}$ & $\begin{array}{l}0.3484 \\
2\end{array}$ & $\begin{array}{l}0.2893 \\
8\end{array}$ & 0.42085 \\
\hline Alpha Wave & $\begin{array}{l}0.050 \\
147\end{array}$ & $\begin{array}{l}0.248 \\
72\end{array}$ & $\begin{array}{l}0.1250 \\
9\end{array}$ & $\begin{array}{l}0.1031 \\
2\end{array}$ & 0.20168 \\
\hline Beta Wave & $\begin{array}{l}0.002 \\
785\end{array}$ & $\begin{array}{l}0.021 \\
714\end{array}$ & $\begin{array}{l}0.0073 \\
007\end{array}$ & $\begin{array}{l}0.0056 \\
305\end{array}$ & $\begin{array}{l}0.01406 \\
6\end{array}$ \\
\hline
\end{tabular}


Table 3: S0 stage analysis of RBD patient EEG data in terms of Energy Level

\begin{tabular}{|l|l|l|l|}
\hline & Case 1 & Case 2 & Case 3 \\
\hline Delta Wave & 0.6866 & 0.5481 & 0.6866 \\
\hline Theta Wave & 0.2377 & 0.2148 & 0.2377 \\
\hline Alpha Wave & 0.0710 & 0.1997 & 0.0710 \\
\hline Beta Wave & 0.0045 & 0.0372 & 0.0045 \\
\hline
\end{tabular}

Table 3 and 4 are here for the S0 stage pattern analysis of the RBD disorder and normal person cases. Again multiple cases are taken here. The energy level on average is observed to be decreased in RBD patients in the alpha wave as compared to the normal person.

Table 4: S0 stage analysis of Normal Person EEG data in terms of Energy Level

\begin{tabular}{|l|l|l|l|}
\hline & Case 1 & Case 2 & Case 3 \\
\hline Delta Wave & 0.4578 & 0.4986 & 0.4375 \\
\hline Theta Wave & 0.3435 & 0.3603 & 0.3126 \\
\hline Alpha Wave & 0.1910 & 0.1355 & 0.1327 \\
\hline Beta Wave & 0.0074 & 0.0055 & 0.0054 \\
\hline
\end{tabular}

\section{CONCLUSION}

In the modern busy world the impact of stress and restless life style is on prominence. This has established a culture of small sleep with high depression and anxiety. Due to heavy workload and challenges at workplace people are getting disorders in sleep activity and suffering from sleeplessness or inadequate sleep. This article has developed an algorithm to diagnose the sleep disorder of RBD type using the energy level at different sleep stages under multiple sleep waves. The approach has fussed out the frequency based classifier strategy to justify the circumstances under which a person is belonging to RBD sleep disorder. In future this approach may be combined with hybrid to the modern artificial intelligence technique to develop an automated model of diagnosis of other sleep disorders.S0 stage has found to be crucial to distinguish the RBD sleep disorder. The average percentage energy level in RBD patient is 0.1139 while in normal cases it is 0.1530 during alpha activity. Hence it is concluded that the percentage energy level is decreased in RBD patients in alpha activity of S0 stage

\section{REFERENCES}

[1] A. M. da Silva Pinho, N. Pombo and N. M. Garcia, "Sleep apnea detection using a feed-forward neural network on ECG signal," 2016 IEEE 18th International Conference on e-Health Networking, Applications and Services (Healthcom), Munich, 2016, pp. 1-6. https://doi.org/10.1109/HealthCom.2016.7749468
[2] V. César CavalcantiRoza, A. M. de Almeida and O. Adrian Postolache, "Design of an artificial neural network and feature extraction to identify arrhythmias from ECG," 2017 IEEE International Symposium on Medical Measurements and Applications (MeMeA), Rochester, MN, 2017, pp. 391-396. https://doi.org/10.1109/MeMeA.2017.7985908

[3] N. Naseer and H. Nazeer, "Classification of normal and abnormal ECG signals based on their PQRST intervals," 2017 International Conference on Mechanical, System and Control Engineering (ICMSC), St. Petersburg, 2017, pp. 388-391. https://doi.org/10.1109/ICMSC.2017.7959507

[4] A. A. S. Raj, N. Dheetsith, S. S. Nair and D. Ghosh, "Auto analysis of ECG signals using artificial neural network," 2014 International Conference on Science Engineering and Management Research (ICSEMR), Chennai, 2014, pp. 1-4.

[5] T. F. Lesmana, S. M. Isa and N. Surantha, "Sleep Stage Identification Using the Combination of ELM and PSO Based on ECG Signal and HRV," 2018 3rd International Conference on Computer and Communication Systems (ICCCS), Nagoya, 2018, pp. 258-262. https://doi.org/10.1109/CCOMS.2018.8463307

[6] Urtnasan, E., Park, J. \& Lee, K. Automatic detection of sleep-disordered breathing events using recurrent neural networks from an electrocardiogram signal. Neural Comput\&Applic 32, 4733-4742 (2020). https://doi.org/10.1007/s00521-018-3833-2

[7] Nishad, Anurag, Ram Bilas Pachori, and U. Rajendra Acharya. "Application of TQWT based filter-bank for sleep apnea screening using ECG signals." Journal of Ambient Intelligence and Humanized Computing (2018): $1-12$. https://doi.org/10.1007/s12652-018-0867-3

[8] Karthik, R., DhruvTyagi, AmoghRaut, SoumyaSaxena, K. P. Bharath, and M. Rajesh Kumar. "Implementation of Neural Network and feature extraction to classify ECG signals." In Microelectronics, Electromagnetics and Telecommunications, pp. 317-326. Springer, Singapore, 2019. https://doi.org/10.1007/978-981-13-1906-8_33

[9] Walia Navneet, Singh Harsukhpreet, Sharma Anurag "ANFIS: Adaptive Neuro-Fuzzy Inference System- A Survey." International Journal of Computer Application, Volume 123-No.13, August 2015. https://doi.org/10.5120/ijca2015905635

[10] Pinho, André, Nuno Pombo, Bruno MC Silva, KouamanaBousson, and Nuno Garcia. "Towards an accurate sleep apnea detection based on ECG signal: The quintessential of a wise feature selection." Applied Soft Computing 83 (2019): 105568.

https://doi.org/10.1016/j.asoc.2019.105568

[11] Pan, Jiapu, and Willis J. Tompkins. "A real-time QRS detection algorithm." IEEE transactions on biomedical engineering 3 (1985): 230-236.

[12] Xie, Baile, and Hlaing Minn. "Real-time sleep apnea detection by classifier combination." IEEE Transactions on information technology in biomedicine 16 , no. 3 (2012): 469-477. https://doi.org/10.1109/TITB.2012.2188299

[13] Lin, Chin-Teng, and Chia-Feng Juang. "An adaptive neural fuzzy filter and its applications." IEEE 
Transactions on Systems, Man, and Cybernetics, Part B (Cybernetics) 27, no. 4 (1997): 635-656. https://doi.org/10.1109/3477.604107

[14] Young Soo Jang, Young Han Kook, Jeong-lae Kim, Hyun-Woo Jeong., "Development of the cost-effective, miniaturized vein imaging system with enhanced noise reduction", International Journal of Advanced Trends in Computer Science and Engineering, Volume 8, No.6, November - December 2019.

https://doi.org/10.30534/ijatcse/2019/80862019
[15] Irma T. Plata, "Development and Testing of Embedded System for Smart Detection and Recognition of Witches Broom Disease on Cassava Plants using Enhanced ViolaJones and Template Matching Algorithm", International Journal of Advanced Trends in Computer Science and Engineering, Volume 8, No.5, September - October 2019. https://doi.org/10.30534/ijatcse/2019/113852019 